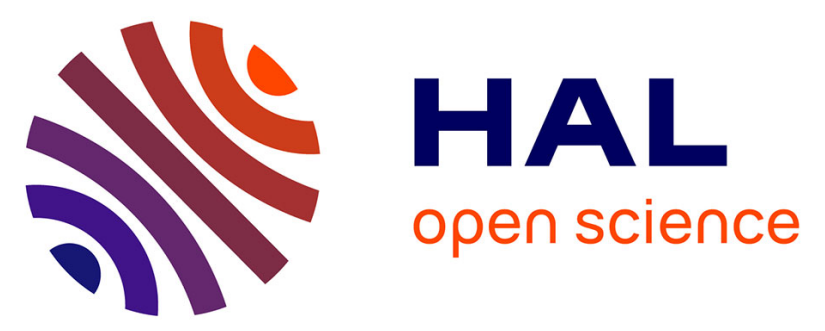

\title{
Recommandations du Collège des enseignants de médecine vasculaire (CEMV) : analyse des flux artériels Doppler (classification de Saint-Bonnet simplifiée)
}

Guillaume Mahé, C Boulon, I Désormais, P Lacroix, L Bressollette, J L Guilmot, C Le Hello, M A Sevestre, G Pernod, Jean-Marc Constans, et al.

\section{To cite this version:}

Guillaume Mahé, C Boulon, I Désormais, P Lacroix, L Bressollette, et al.. Recommandations du Collège des enseignants de médecine vasculaire (CEMV) : analyse des flux artériels Doppler (classification de Saint-Bonnet simplifiée). JMV-Journal de Médecine Vasculaire, 2018, 43 (4), pp.255-261. 10.1016/j.jdmv.2018.05.002 . hal-01862282

\section{HAL Id: hal-01862282}

https://hal-univ-rennes1.archives-ouvertes.fr/hal-01862282

Submitted on 10 Sep 2018

HAL is a multi-disciplinary open access archive for the deposit and dissemination of scientific research documents, whether they are published or not. The documents may come from teaching and research institutions in France or abroad, or from public or private research centers.
L'archive ouverte pluridisciplinaire HAL, est destinée au dépôt et à la diffusion de documents scientifiques de niveau recherche, publiés ou non, émanant des établissements d'enseignement et de recherche français ou étrangers, des laboratoires publics ou privés. 
2 Recommandations du Collège des Enseignants de Médecine Vasculaire (CEMV) : analyse des

3 flux artériels Doppler (Classification de Saint-Bonnet simplifiée).

4

5 CEMV (College of the French Vascular Medicine Teachers) statement: Arterial Doppler

6 waveforms analysis (Simplified Saint-Bonnet Classification)

7 G. Mahé a

8 C. Boulon b

9 I. Désormais c

10 P. Lacroix C

11 L. Bressollette d

12 J.L. Guilmot e

13 C. Le Hello f

14 M.A. Sevestre g

15 G. Pernod h J.

16 Constans b C.

17 Boissier $\mathrm{f}$ A.

18 Bura-Rivière i

19 Collège des enseignants de médecine vasculaire (CEMV)

20

21 a

22 Inserm, CIC 1414, univ Rennes, CHU Rennes, unité de médecine vasculaire, 35000 Rennes, France 23

$24 \quad b$

25 Médecine vasculaire, hôpital Saint-André, 33076 Bordeaux, France 
28 Médecine vasculaire, CHU Limoges, 87042 Limoges, France

29

$30 \mathrm{~d}$

31 Médecine vasculaire, CHU de Brest, 29200 Brest, France

32

33

e

34 Médecine vasculaire, 37044 Tours, France

35

36

37

Service de médecine vasculaire et thérapeutique, CHU Nord Saint-Etienne, 42055 Saint-Étienne,

38 France

39

$40 \mathrm{~g}$

41 Médecine vasculaire, CHU Amiens, 80054 Amiens, France

42

$43 \mathrm{~h}$

44 Médecine vasculaire, CHU de Grenoble, 38700 La Tronche, France

45

$46 \quad \mathrm{i}$

47 Médecine vasculaire, hôpital Rangueil, CHU de Toulouse, 31059 Toulouse, France

48

49 Résumé : L’artériopathie des membres inférieurs est une pathologie fréquente. L’analyse des flux

50 artériels Doppler est un élément important pour juger de l'état hémodynamique d'un patient et

51 affirmer la présence ou non d'une artériopathie des membres inférieurs. Malheureusement, à ce

52 jour, il existe une grande hétérogénéité dans la description de l'analyse des flux Doppler. Afin de 
palier cette problématique, la classification de Saint-Bonnet va être enseignée aux capacitaires et aux futurs internes de la discipline afin d’homogénéiser nos pratiques.

Mots clés : Artériopathie des membres inférieurs ; Flux Doppler ; Diagnostic

Summary: Lower extremity peripheral artery disease is a frequent disease. Arterial Doppler waveforms analysis is a key element in vascular medicine especially to diagnose lower peripheral artery disease. Although Doppler waveforms are often used, a high heterogeneity exists about the description of the arterial Doppler waveforms. This review presents the simplified Saint-Bonnet classification that is tought to vascular medicine residents in order to homogenize arterial flow description.

Keywords: Peripheral artery disease; Diagnosis; Doppler, Waveforms

\section{Introduction}

L’artériopathie oblitérante des membres inférieurs (AOMI) est une pathologie fréquente qui touche plus de 200 millions de personnes dans le monde et qui est principalement d'origine athéromateuse [1]. La prévalence de cette pathologie est estimée en France à $11 \%$ chez les plus de 40 ans [2].

Le diagnostic d’AOMI peut être fait par la mesure de l'index de pression systolique de cheville mais aussi par l'analyse des flux Doppler [3,4].

Toutes les recommandations internationales s'accordent pour dire que l’index de pression systolique (IPS) de cheville de repos est la technique de référence pour diagnostiquer une AOMI [3,5,6]. Cependant, dans certaines populations comme chez les diabétiques, les insuffisants rénaux, et les personnes âgées notamment, l’IPS de repos peut être faussement normal [5]. Dans ces cas et compte tenu des difficultés d'apprentissage, l'évaluation des flux Doppler peut apparaître comme intéressante [7-10]. Ainsi, l'artériopathie des membres inférieurs peut être définie au repos $\operatorname{par}[3,6,11]$ : 
-Un IPS de repos $\leq 0,90$ et un flux Doppler « anormal »

-Un IPS de repos $\leq 0,90$ et un flux Doppler normal (triphasique)

-Un IPS de repos > 0,90 et un flux Doppler « anormal »

La grande difficulté d'utiliser l'analyse des flux Doppler est liée au fait que la description/dénomination des différents flux artériels Doppler est à ce jour très hétérogène [4,12]. Cette inhomogénéité pose des problèmes à plusieurs niveaux : dans la pratique clinique où selon les médecins la dénomination n’est pas la même, rendant difficiles les échanges avec nos différents confrères; dans l'enseignement où selon que l'on ait été formé à tel ou tel endroit les dénominations varient; et dans la recherche où les comparaisons entre les études sur les flux sont difficilement comparables car elles n’utilisent pas les mêmes classifications.

Pour répondre à ces difficultés et dans le cadre de la réforme pédagogique avec la naissance du Diplôme d’Etudes Spécialisées (DES) en Médecine Vasculaire, il nous est apparu indispensable de définir des termes couramment employés lors de notre pratique de l'écho-Doppler et de proposer une classification simple et rapide pour décrire les flux artériels Doppler des membres inférieurs [4]. Ce travail présente d'abord les dénominations des différents flux, puis la classification de SaintBonnet simplifiée puis les facteurs influençant le flux Doppler et enfin les recommandations pour l’analyse des flux et les moyens pédagogiques à mettre en œuvre.

1) Définitions et dénominations :

a) Flux artériel normal de repos :

Dans une artère saine des membres inférieurs au repos, le flux artériel est laminaire avec un caractère pulsatile. Le morphotype vélocimétrique normal présente différents aspects aussi appelés « modulations » décrites de la façon suivante (Figure 1):

1) une branche ascendante (branche anacrote) et son temps de montée (court),

2) une branche descendante (branche catacrote) et son temps de descente (court),

3) une composante diastolique négative, 
4) un rebond diastolique positif,

5) un retour à la ligne de base.

En plus de ces différentes phases, le morphotype vélocimétrique normal inclut une fenêtre spectrale, qui doit être claire, ce qui signifie que l'ensemble des globules rouges se déplace à une vitesse quasi similaire. A contrario, lorsque la fenêtre spectrale n'est plus claire, cela signifie que les globules rouges ne se déplacent plus tous à la même vitesse.

b) Flux démodulé :

112 Un flux démodulé se traduit par une perte de modulation caractérisée par une perte du caractère triphasique (Figure 2). c) Flux amorti :

Un flux amorti se traduit par un morphotype vélocimétrique dont le temps de montée systolique est allongé c'est-à-dire avec un temps de montée systolique $>70$ ms ou à 100 ms suivant les auteurs 117 (Figure 2).

d) Flux ralenti :

Un flux ralenti se traduit par un morphotype vélocimétrique comportant un temps de montée systolique normal mais une vitesse maximale systolique diminuée (Figure 2).

2) Classification de Saint-Bonnet simplifiée (Figure 3) :

Différents classifications ont déjà été proposées mais elles ne permettent pas de classer l'ensemble des flux [13,14]. La classification de Saint-Bonnet basée sur les classifications précédentes et les travaux de F. Becker permet de résoudre cette difficulté [11-13]. Il existe deux classifications de Saint-Bonnet : la classification classique et la classification simplifiée [4]. Seule la classification simplifiée est présentée dans cette mise au point car elle est plus simple d’utilisation (Figure 3). La courbe Doppler comporte successivement : une branche ascendante, une branche descendante, 129 suivie d’une composante diastolique négative, précédent un rebond diastolique positif avant un 
130 131 être continu (sans retour à la ligne de base).

132 L'analyse objective d'un morphotype vélocimétrique triphasique porte sur la branche ascendante 133 (branche anacrote) avec un temps de montée court, la branche descendante (branche catacrote) avec

retour à la ligne de base. En présence d’une diminution des résistances périphériques le flux peut

un temps de descente court, la composante diastolique négative, le rebond diastolique positif et le retour à la ligne de base (stade Saint-Bonnet $\mathrm{N}$ ). Les altérations du signal vélocimétrique comportent successivement en fonction de la sévérité des lésions artérielles :

- la disparition du rebond diastolique positif (morphotype vélocimétrique biphasique, stade SaintBonnet A),

- la disparition de la composante diastolique négative (morphotype vélocimétrique monophasique symétrique avec pic systolique pointu, stade Saint-Bonnet B),

- l'augmentation du temps de descente de la branche catacrote (morphotype vélocimétrique monophasique asymétrique avec pic systolique « émoussé » avec une branche descendante dont la pente est diminuée, ou l'augmentation du temps de montée systolique de la branche anacrote avec présence d’un pic systolique « émoussé » (stade Saint-Bonnet CD),

- l'effondrement des vitesses systoliques avec une augmentation du temps de montée systolique et une augmentation du temps de descente de la branche catacrote (stade Saint-Bonnet E),

- absence de flux (stade Saint-Bonnet O).

Si le signal ne revient pas à la ligne de base, le flux est dit continu (fc) et peut être rencontré à tous les stades de la classification (sauf pour le stade Saint-Bonnet O). Pour un stade B à flux continu, le flux est alors noté « stade B-fc » par exemple. La composante «fc » est liée à l'état vasculaire en aval de la zone d'enregistrement. En cas d’ischémie par exemple et sous réserve que le système vasculaire du patient ait conservé sa capacité de vasodilatation, la composante «fc » sera présente. Cette composante «fc » peut aussi être retrouvée lors de processus inflammatoires locaux ou lors de l'exercice. 
155 Finalement à ces stades s’ajoutent deux stades qui ne sont pas liés à la sévérité de la maladie 156 athéromateuse à savoir le flux rencontré dans les faux-anévrismes (Saint-Bonnet FA) et le flux 157 Saint-Bonnet U (U pour Undefined) qui est à utiliser quand le flux visualisé ne correspond à aucun 158 flux présenté précédemment de la classification (Figure 3). Le flux Saint-Bonnet U peut être 159 retrouvé lors de dissection artérielle par exemple où l’on peut observer des flux très atypiques.

\section{3) Facteurs influençant la forme du flux Doppler :}

162 La forme du flux va être modifiée en fonction de la présence ou non de lésion sur l'arbre artériel mais aussi en présence de thérapeutiques ou de phénomène inflammatoire par exemple. Il est classique en Doppler d’étudier le flux au niveau de la lésion artérielle ce qui définit les signes directs et d'étudier le retentissement en amont et en aval ce qui définit les signes indirects d'amont et les signes indirects d'aval. La figure 4 présente les modifications du flux Doppler en fonction du degré de sténose. Le tableau 1 présente l’ensemble des possibilités attendues avec la classification de Saint Bonnet.

4) Recommandations et suggestions (Tableau 2) :

171 Nous recommandons de réaliser ces mesures de flux après 5 à 10 minutes de repos dans une pièce 172 où le patient sera en neutralité thermique en décubitus (CdR I, NdP C).

174 Les enregistrements des flux artériels au Doppler peuvent être réalisés au repos. En cas de flux normaux, chez un patient avec une forte suspicion d'AOMI, nous recommandons de réaliser une exploration des flux artériels après effort, afin de sensibiliser le diagnostic et ce pour les mêmes raisons que la réalisation d’un index de pression systolique post-effort (CdR I, NdP C). 
179 Nous recommandons d’analyser les morphotypes vélocimétriques artériels chez tous les patients qui 180 doivent bénéficier d'une mesure de l'index de pression systolique de repos que les patients soient 181 symptomatiques ou non. Ces données doivent figurer sur le compte rendu (CdR I, NdP C).

183 Nous recommandons d'effectuer les enregistrements du flux artériel et d'analyser les morphotypes 184 vélocimétriques recueillis tout au long de l'arbre artériel (aorte, artères iliaque commune, iliaque 185 externe, fémorale commune, fémorale superficielle, poplitée, artères tibiale postérieure, pédieuse 186 (dorsale du pied) et fibulaire) et de notifier cette information dans le compte rendu (CdR I, NdP C).

Nous recommandons d'effectuer les enregistrements du flux artériel et d'analyser les morphotypes vélocimétriques dans tous les pontages et matériels endovasculaires et de notifier ces informations dans le compte rendu (CdR I, NdP C).

Nous recommandons de réaliser les enregistrements post-effort le plus tôt après l'arrêt de l'effort et de noter le temps entre l'arrêt de l'effort et le moment de l'enregistrement. Pour simplifier la mesure post-effort, il est conseillé de marquer au feutre lorsque le patient est au repos la zone d'enregistrement. Le type d'effort ainsi que les symptômes présentés par le patient doivent être rapportés (CdR I, NdP C).

Les personnes réalisant des explorations vasculaires doivent être entraînées à l'analyse des courbes Doppler (CdR I, NdP C).

201 Nous recommandons d'utiliser une classification de Saint-Bonnet simplifiée pour décrire les flux 
205 Compte tenu de l'importante variabilité des classifications des morphotypes vélocimétriques 206 artériels enregistrés par méthode Doppler [15], il apparaît nécessaire de s’intéresser aux méthodes 207 d’enseignement. Aucune méthode particulière n’a été proposée ni validée pour l'enseignement de 208 l'enregistrement des signaux Doppler alors même que cette analyse du signal Doppler recueilli est 209 un élément important de la pratique vasculaire. Un enseignement théorique, comme réalisé dans la 210 plupart des facultés françaises de Médecine pour la mesure de l’IPS de repos, serait probablement 211 inapproprié [16]. Pour l'IPS, il est montré qu’il faut réaliser par exemple un certain nombre de 212 mesures pour devenir compétent [7,8,17]. Les travaux de Scissons montrent une meilleure 213 classification des morphotypes vélocimétriques avec l'augmentation de l'expérience de la personne 214 analysant les flux [15,18]. Des études méritent d’être menées pour identifier la meilleure manière 215 d'enseigner l'analyse des flux artériels et ainsi améliorer les pratiques pour mieux prendre en charge 216 les patients.

\section{Conclusion}

219 L’analyse du morphotype vélocimétrique recueilli par méthode Doppler est un moyen « ancien », 220 simple, peu coûteux et sans risque pour évaluer l’hémodynamique artérielle. Il n’a cependant jamais 221 fait l'objet d'un consensus. La classification de Saint-Bonnet dont l'utilisation est suggérée par les 222 enseignants du Collège des Enseignants de Médecine Vasculaire permet de standardiser cette pratique. 
230 Tableau 1: Principales modifications attendues du flux artériel en fonction du degré de

231 rétrécissement artériel avec la classification de Saint-Bonnet simplifiée.

\begin{tabular}{|c|c|c|c|c|c|}
\hline Degré de rétrécissement & $<40 \%$ & 40 et $70 \%$ & $>70-75 \%$ & $>90 \%$ & Occlusion \\
\hline $\begin{array}{l}\text { Flux en amont du } \\
\text { rétrécissement }\end{array}$ & $\mathrm{N}$ & $\mathrm{N}$ & $\mathrm{N}$ ou $\mathrm{A}$ & $\begin{array}{l}\mathrm{N} \text { ou } \mathrm{A} \text { avec } \\
\text { vitesse } \\
\text { diminuée }\end{array}$ & $\begin{array}{l}\mathrm{N} \text { ou A avec } \\
\text { vitesse } \\
\text { diminuée }\end{array}$ \\
\hline $\begin{array}{l}\text { Flux au niveau du } \\
\text { rétrécissement }\end{array}$ & $\mathrm{N}$ & $\begin{array}{c}\mathrm{N} \text { avec } \\
\text { vitesse } \\
\text { augmentée }\end{array}$ & $\begin{array}{l}\text { A-fc ou B-fc } \\
\text { avec vitesse } \\
\text { augmentée }\end{array}$ & $\begin{array}{l}\text { A-fc ou B-fc } \\
\text { avec vitesse } \\
\text { augmentée }\end{array}$ & 0 \\
\hline $\begin{array}{l}\text { Flux en aval du } \\
\text { rétrécissement en } \\
\text { l'absence de collatérale }\end{array}$ & $\mathrm{N}$ & $\mathrm{N}$ & $\begin{array}{l}\text { CD-fc ou CD } \\
\text { ou E-fc ou E }\end{array}$ & $\begin{array}{l}\text { CD-fc ou CD } \\
\text { ou E-fc ou E }\end{array}$ & 0 \\
\hline $\begin{array}{l}\text { Flux en aval du } \\
\text { rétrécissement avec } \\
\text { collatérale efficace au } \\
\text { repos }\end{array}$ & & & $\mathrm{N}$ & $\mathrm{N}$ & $\mathrm{N}$ \\
\hline $\begin{array}{l}\text { Flux en aval du } \\
\text { rétrécissement avec } \\
\text { collatérale non efficace à } \\
\text { l'effort }\end{array}$ & & & $\begin{array}{l}\text { CD-fc ou CD } \\
\text { ou E-fc ou E }\end{array}$ & $\begin{array}{l}\text { CD-fc ou CD } \\
\text { ou E-fc ou E }\end{array}$ & $\begin{array}{l}\text { CD-fc ou CD } \\
\text { ou E-fc ou E }\end{array}$ \\
\hline
\end{tabular}

233 Tableau 1 légende : fc signifie flux continu. Il est important de noter que ces modifications sont les

234 modifications attendues mais qu’elles n’ont pas été validées par des études 
Tableau 2 : Classe de recommandations et niveaux de preuve

Classe de recommandations Définition Phrase à utiliser

Classe I

Preuve que la procédure est efficace, Est recommandé, est indibénéfique, utile qué

Classe II

Preuve conflictuelle ou divergence

d’opinion sur l'efficacité ou l'utilité de

la procédure

Classe IIa

Poids des preuves/opinion est en faveur de l'efficacité/utilité.

Devrait être considéré

Classe IIb L’utilité/efficacité est moins bien établie par les preuves

Peut être considéré

Preuve que la procédure n'est pas utile

Classe III ou efficace, et qu'elle peut être né-

N’est pas recommandée faste.

Niveau de Preuve

Niveau de Preuve A

Niveau de Preuve B

Niveau de Preuve C
Justification

Les données proviennent de multiples essais randomisés ou métaanalyses.

Les données viennent d'un seul essai randomisé ou de larges études non randomisées.

Consensus d'opinion d'experts ou petites études rétrospectives, registres. 
260 Figure 1 : Description d'un flux Doppler artériel normal.

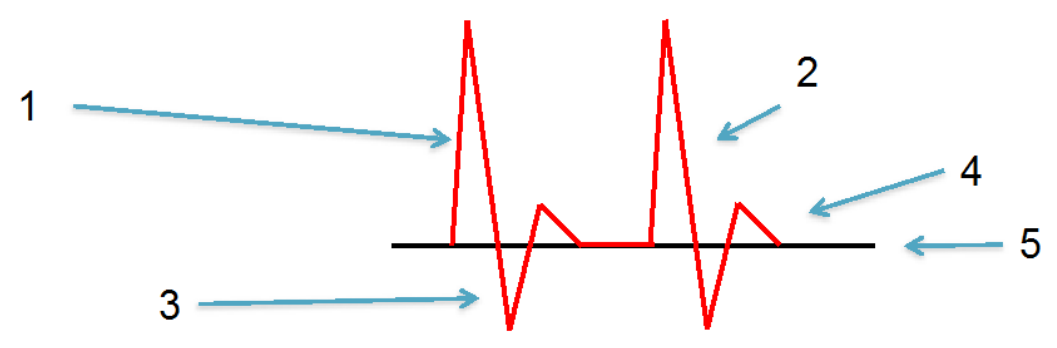

Le morphotype vélocimétrique normal (Saint-Bonnet $N$ ) est triphasique, il comporte :

-Une branche ascendante rapide (1)

-Une branche descendante rapide (2)

-Une composante diastolique négative (3)

-Un rebond diastolique (4)

-II se termine au niveau de la ligne de base (5) 
274 Figure 2 : Définitions pour l'analyse des flux Doppler.

Modulation

Flux normalement modulé

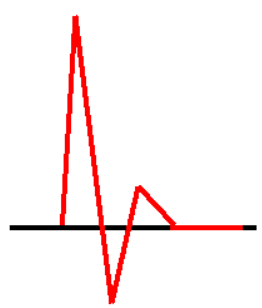

Flux démodulés
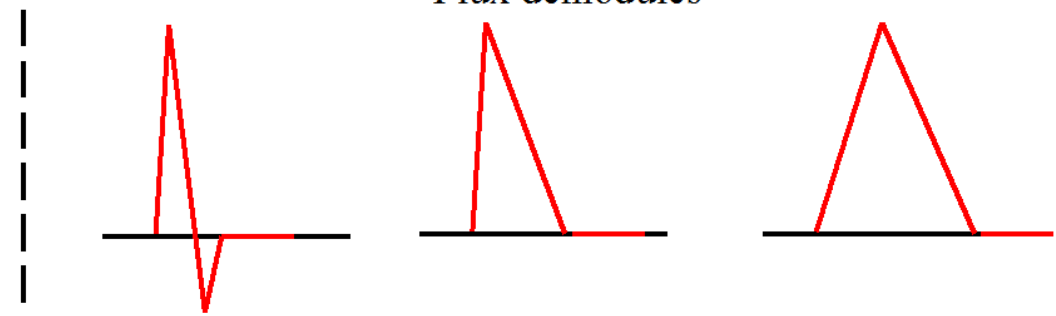

Amortissement
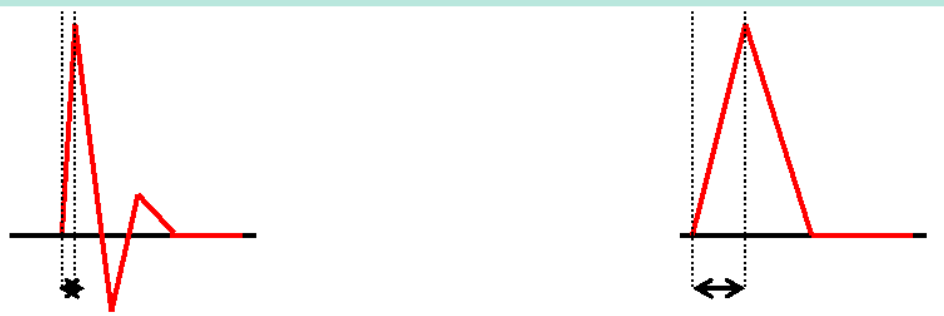

Ralentissement

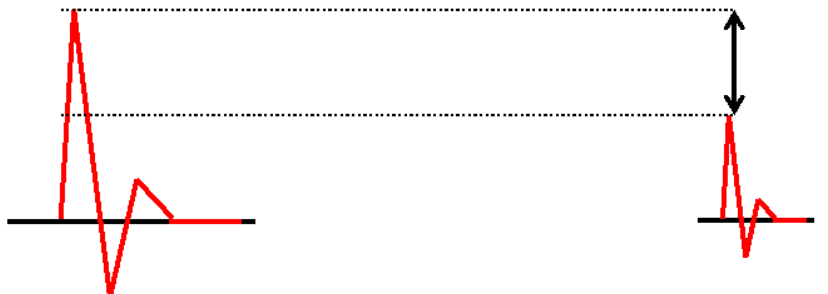


Figure 3 : Classification de Saint-Bonnet simplifiée.

A) Classification de Saint-Bonnet simplifié sans flux continu

$\mathrm{N}$

A

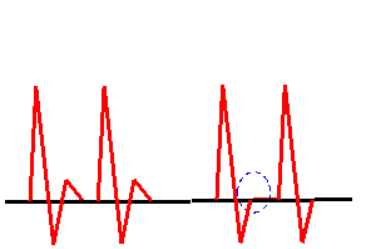

B

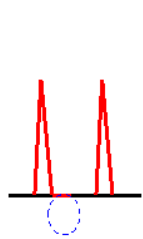

CD

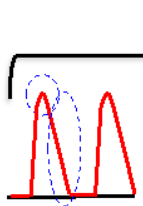

$E$

0

Classification de Saint-Bonnet simplifié avec flux continu
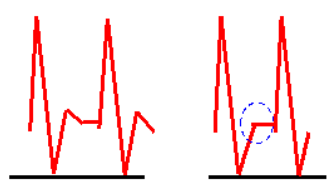

$\mathrm{N}-\mathrm{cf}$
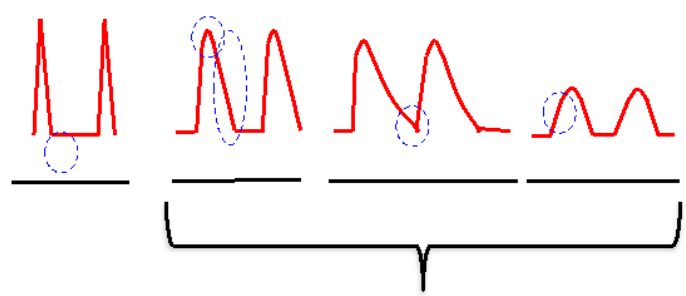

B-cf

CD-cf

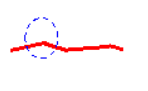

E-cf 0

B)

Flux de faux-anévrisme : Saint-Bonnet FA

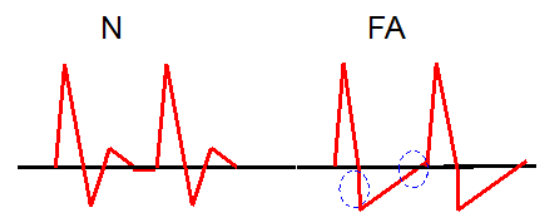

Flux indéfini: Saint-Bonnet U

Dans certains cas, les flux ne correspondent à aucune description précédente, il convient alors de les décrire comme flux indéfini noté Saint-Bonnet $U$ 

artériel.

A)
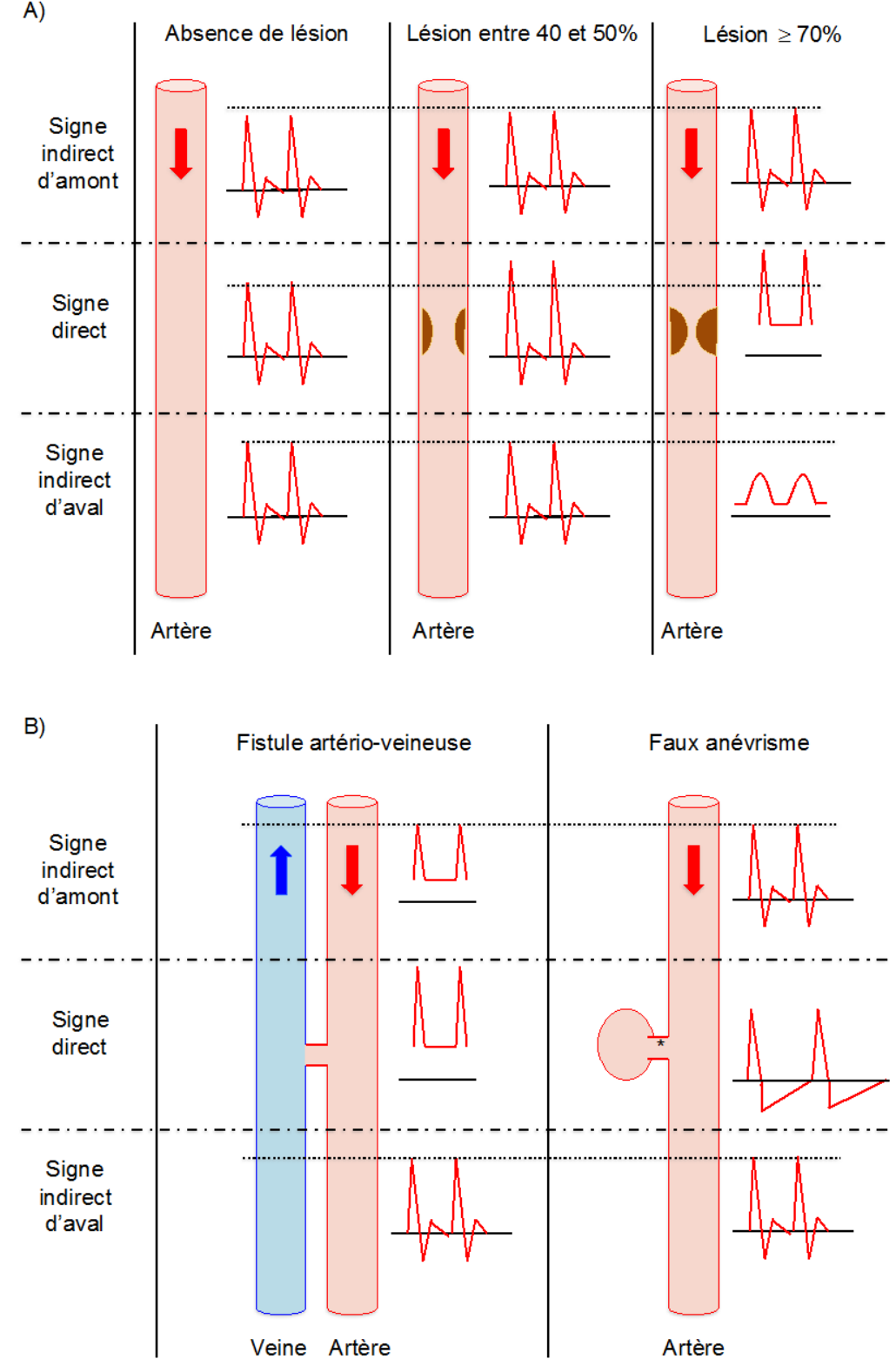

Figure 4 légende : A) modification du flux en fonction du degré de rétrécissement artériel ; A noter qu'en présence d'une lésion $\geq 70 \%$ le flux en amont peut être A et qu'au niveau de la sténose d'autres types de flux peuvent être retrouvés (cf. Tableau 1). B) Modification du flux en présence d'une fistule artério-veineuse et d'un faux anévrisme ; * indique le lieu de la mesure du flux Doppler. 
Références :

[1] Fowkes FGR, Rudan D, Rudan I, Aboyans V, Denenberg JO, McDermott MM, et al. Comparison of global estimates of prevalence and risk factors for peripheral artery disease in 2000 and 2010: a systematic review and analysis. Lancet Lond Engl 2013;382:1329-40. doi:10.1016/S0140-6736(13)61249-0.

[2] Boccalon H, Lehert P, Mosnier M. [Assessment of the prevalence of atherosclerotic lower limb arteriopathy in France as a systolic index in a vascular risk population]. J Mal Vasc 2000;25:38-46.

[3] Gerhard-Herman MD, Gornik HL, Barrett C, Barshes NR, Corriere MA, Drachman DE, et al. 2016 AHA/ACC Guideline on the Management of Patients With Lower Extremity Peripheral Artery Disease: A Report of the American College of Cardiology/American Heart Association Task Force on Clinical Practice Guidelines. Circulation 2017;135:e726-79. doi:10.1161/CIR.0000000000000471.

[4] Mahé G, Boulon C, Desormais I, Lacroix P, Bressollette L, Guilmot J-L, et al. Statement for Doppler waveforms analysis. VASA Z Gefasskrankheiten 2017:1-9. doi:10.1024/03011526/a000638.

[5] Aboyans V, Criqui MH, Abraham P, Allison MA, Creager MA, Diehm C, et al. Measurement and interpretation of the ankle-brachial index: a scientific statement from the American Heart Association. Circulation 2012;126:2890-909. doi:10.1161/CIR.0b013e318276fbcb.

[6] Mahé G, Jaquinandi V. [Diagnosis of lower limb peripheral artery disease]. Presse Medicale Paris Fr 1983 2017. doi:10.1016/j.lpm.2017.09.021.

[7] Chaudru S, de Müllenheim P-Y, Le Faucheur A, Kaladji A, Jaquinandi V, Mahé G. Training to Perform Ankle-Brachial Index: Systematic Review and Perspectives to Improve Teaching and Learning. Eur J Vasc Endovasc Surg Off J Eur Soc Vasc Surg 2016;51:240-7. doi:10.1016/j.ejvs.2015.09.005.

[8] Chaudru S, de Müllenheim P-Y, Le Faucheur A, Jaquinandi V, Kaladji A, Mahe G. Knowledge about ankle-brachial index procedure among residents: being experienced is beneficial but is not enough. VASA Z Gefasskrankheiten 2016;45:37-41. doi:10.1024/03011526/a000493.

[9] Donnou C, Chaudru S, Stivalet O, Paul E, Charasson M, Selli J-M, et al. How to become proficient in performance of the resting ankle-brachial index: Results of the first randomized controlled trial. Vasc Med 2017:1358863X1774099. doi:10.1177/1358863X17740993.

[10] Donnou C, Chaudru S, Stivalet O, Paul E, Charasson M, Selli J-M, et al. Medical students’ proficiency in performance of the resting ankle-brachial index is not sustained at 6 months. $\mathrm{J}$ Clin Hypertens Greenwich Conn 2018. doi:10.1111/jch.13206.

[11] Becker F, Luizy F, Baud J-M, Pichot O, SFMV. [Quality standards for ultrasound assessment (CW-Doppler, Duplex US) of the lower limb arteries in vascular medicine. Report of the French Society for Vascular Medicine]. J Mal Vasc 2011;36:364-85. doi:10.1016/j.jmv.2011.10.002.

[12] Omarjee L, Stivalet O, Hoffmann C, Scissons R, Bressollette L, Mahé G, et al. Heterogeneity of Doppler waveforms description is decreased with the use of a dedicated classification. VASA Z Gefasskrankheiten 2018:Accepted.

[13] Descotes J, Cathignol D. [Classification of changes in circulatory rate in the arteries of the lower limbs. Transcutaneous measurement by Doppler effect]. Nouv Presse Med 1975;4:2091-3. 
[14] Spronk S, den Hoed PT, de Jonge LCW, van Dijk LC, Pattynama PMT. Value of the duplex waveform at the common femoral artery for diagnosing obstructive aortoiliac disease. J Vasc Surg 2005;42:236-42; discussion 242. doi:10.1016/j.jvs.2005.04.048.

[15] Scissons, Robert. Characterizing triphasic, biphasic, and monophasix Doppler waveforms: Should a simple task be so difficult? J Diagn Med Sonogr 2008:269-76.

[16] Mahé G. [Ankle-brachial index measurement: Methods of teaching in French medical schools and review of literature]. J Mal Vasc 2015;40:165-72. doi:10.1016/j.jmv.2015.03.001.

[17] Georgakarakos E, Papadaki E, Vamvakerou V, Lytras D, Tsiokani A, Tsolakaki O, et al. Training to measure ankle-brachial index at the undergraduate level: can it be successful? Int J Low Extrem Wounds 2013;12:167-71. doi:10.1177/1534734613483768.

[18] Scissons, Robert, Comerota, AJ. Confusion of peripheral arterial Doppler waveform terminology. J Diagn Med Sonogr 2009:189-94. 